\title{
O processo de trabalho em uma Unidade de Terapia Intensiva Neonatal
}

THE WORKING PROCESS OF A NEONATAL INTENSIVE CARE UNIT

EL PROCESO DE TRABAJO EN UNA UNIDAD DE TERAPIA INTENSIVA NEONATAL

Patricia de Araújo Marques ${ }^{1}$, Enirtes Caetano Prates Melo²

\section{RESUMO}

Este estudo teve como objetivo analisar a organização do processo de trabalho em uma Unidade de Terapia Intensiva Neonatal através das relações estabelecidas entre os atores sociais presentes no espaço micropolítico. Trata-se de uma metodologia não-convencional, adaptada a estudo qualitativo. Adotou-se um estudo de série de casos cuja trajetória foi denominada trajetória-sentinela e, através desta foram, detectados ruídos que influenciaram na qualidade da assistência. A análise do fluxograma revelou fragilidades na rede de contratualidade, problemas de registros e arquivamento de documentação e, erros relativos ao processo assistência.

\section{DESCRITORES}

Enfermagem neonatal

Unidades de Terapia Intensiva Neonatal

Qualidade da assistência à saúde

Avaliação de serviços de saúde

\begin{abstract}
The objective of this study was to analyze the organization of the working process of a Neonatal Intensive Care Unit through the relationships established between the social actors present in the micropolitical space. This study used an unconventional methodology, adapted to a qualitative study. A case series study was adopted, whose trajectory was referred to as sentinel-trajectory, through which it was possible to detect noises that affected the quality of the care provided. The analysis of the flowchart revealed weaknesses of the contract network, problems regarind the registers and documentations, and errors in the health care process.
\end{abstract}

\section{DESCRIPTORS}

Neonatal nursing

Intensive Care Units, Neonatal

Quality of health care

Health services evaluation

\section{RESUMEN}

Este estudio tuvo como objetivo analizar la organización del proceso de trabajo en una Unidad de Terapia Intensiva Neonatal, mediante las relaciones establecidas entre los actores sociales presentes en el espacio micropolítico. Se trata de una metodología no convencional, adaptada al estudio cualitativo. Se adoptó un estudio de serie de casos, cuya trayectoria fue denominada trayectoria-centinela y, a través de ella, se detectaron ruidos que influenciaron en la calidad de la atención. El análisis del flujograma reveló fragilidad en la de de contractualidad, problemas de registro y archivo de documentación, y errores referidos al proceso de atención.

\section{DESCRIPTORES}

Enfermería neonatal

Unidades de Terapia Intensiva Neonatal

Calidad de la atención de salud

Evaluación de servicios de salud

${ }^{1}$ Mestranda do Programa de Pós-Graduação em Enfermagem da Universidade Federal do Estado do Rio de Janeiro. Enfermeira do Instituto Fernandes Figueiras da Fundação Oswaldo Cruz. Rio de Janeiro, RJ, Brasil. enfpmarques@gmail.com ${ }^{2}$ Enfermeira. Doutora em Ciências. Professora Adjunta do Departamento de Enfermagem em Saúde Pública da Escola de Enfermagem Alfredo Pinto da Universidade Federal do Estado do Rio de Janeiro. Rio de Janeiro, RJ, Brasil. enirtes@globo.com

$\begin{aligned} \text { Rev Esc Enferm USP } & \text { Recebido: 22/12/2008 } \\ \text { 2011; 45(2):374-80 } & \text { Aprovado: 12/08/2010 }\end{aligned}$




\section{INTRODUÇÃO}

Na experiência cotidiana de assistência a recém-nascidos de alto risco observa-se que a disponibilidade de recursos considerados essenciais pelo senso comum, tais como estrutura física em conformidade com padrões técnicos, material permanente e de consumo em quantidade e qualidade adequadas e, uma equipe treinada e atualizada, não são suficientes para garantir a qualidade do cuidado prestado ${ }^{(1-2)}$. Há componentes relacionados aos processos de trabalho, não identificados claramente no dia-a-dia, que de fato interferem no produto final do trabalho em saúde, o cuidado. Um algo mais que agrega tanto o componente técnico como as (inter) relações que se estabelecem na organização do processo de trabalho e que, se não compromete a qualidade da assistência, pelo menos afeta sua efetividade.

O cotidiano institucional se expressa, assim, como uma dobra (do sentido e do sem sentido), tanto nos planos individuais como nos coletivos, guardando particularidades. Há, assim, no desempenho da ação processos ruidosos que operam em cada singularidade, bem como entre elas. Tais ruídos representam obstáculos visíveis e invisíveis, tensões, contradições, estranhamentos no processo de trabalho ${ }^{(3-4)}$. De forma análoga à sociedade, há nas instituições de saúde interações de diversas ordens - econômicas, psíquicas, culturais, políticas, éticas, emocionais - que configuram um sistema. Cada sistema traz consigo complementaridades e antagonismos reguladores de enormes perturbações que, em vez de destruí-lo, o mantêm vivo e em funcionamento ${ }^{(5)}$.

O processo de trabalho nas instituições de saúde envolve sujeitos com necessidades de agir e sentir, com visões particulares do mundo. Tratase de um trabalho vivo e, por natureza, não previsível ${ }^{(3,6)}$. A análise do espaço micropolítico permite perceber como se dão as relações trabalhador versus trabalhador e trabalhador versus cliente. Neste lócus de enfrentamentos estão agregados tanto o componente técnico quanto as (inter)relações que se estabelecem entre os atores presentes no ato de cuidar. Logo, se temos um cotidiano tecnicamente satisfatório e ainda assim repleto de tensões é importante lançar um olhar crítico sobre as relações que se processam a nível micropolítico, com o intuito de (re)construir toda a rede que se estabelece na produção do cuidado. Trata-se de uma cartografia da tensão e dos estranhamentos presentes nas amarras das redes sociais das instituições.

No caso específico das unidades de terapia intensiva neonatal, o processo de trabalho é permeado adicionalmente por uma série de particularidades relacionadas à assistência a recém-natos gravemente enfermos. Dentre tais particularidades destaca-se: o uso de uma abordagem

diagnóstica e terapêutica quase sempre invasiva e agressiva; a freqüente introdução de inovações tecnológicas; o estreito limiar entre as respostas favoráveis e possíveis reações adversas à terapia implementada; a imaturidade de vários sistemas orgânicos dos recém-natos, principalmente os prematuros, que pode limitar as respostas fisiológi$\operatorname{cas}^{(7)}$. Neste sentido, o padrão de cuidados requer a utilização de modelos tecno-assistenciais e o envolvimento de uma equipe interdisciplinar qualificada para estabilização das condições do recém-nato.

O processo avaliativo pode funcionar como um instrumento de gestão capaz de se desdobrar em intervenções assistenciais que privilegiem a garantia da qualidade do cuidado, e descrever experiências diversas que agregam obstáculos visíveis e invisíveis. Avaliar, no entanto, não é em geral uma tarefa fácil, uma vez que requer estranhar tudo aquilo que nos é familiar, que faz parte do cotidiano assistencial. Identificar a tensão e os estranhamentos existentes entre o duplo instituído/instituinte requer um distanciamento e um tempo nem sempre disponível entre as equipes de trabalho.

O processo de trabalho nas instituições de saúde sujeitos com sentir, com visões abalho vivo e, por natureza, não previsível.

\section{OBJETIVO}

Analisar o processo de trabalho em uma Unidade de Terapia Intensiva Neonatal (UTIN), tendo como foco principal as relações que se processam no espaço micropolítico da produção do cuidado.

\section{MÉTODO}

Trata-se de um estudo avaliativo com abordagem qualitativa que busca retratar a realidade e compreender a dinâmica das situações, os contrastes e comparações ${ }^{(8)}$. A compreensão dos fenômenos e dos processos sociais traz para análise o subjetivo e o objetivo, o sistema de valores e crenças dos atores implicados, os fatos e seus significados, a ordem e os conflitos.

O cenário da pesquisa foi uma UTIN de uma maternidade de alto risco localizada no Município do Rio de Janeiro. A pesquisa foi desenvolvida em duas etapas; a primeira compreendeu a construção de três trajetórias-sentinelas, derivadas de dois estudos de caso e de uma entrevista.

Na primeira etapa foram estudados dois casos considerados exemplares em função do conjunto de intervenções e procedimentos que demandaram durante o período de internação na instituição estudada. Na seleção dos casos foram incluídos apenas recém-nascidos com idade gestacional abaixo de 32 semanas e peso ao nascer inferior a 1.500 gramas, internados no período compreendido entre janeiro de 2007 a abril de 2008. Neste tipo de delineamento não cabe a utilização de amostragem probabilística, uma vez que o estudo de um único caso já possibi- 
lita a construção do objeto e de suas características essenciais. O estudo retrospectivo dos casos deu origem a três fluxogramas analisadores que permitiram ordenar cronologicamente os fatos e favoreceram a identificação de ruídos relacionados à micropolítica do processo de trabalho. Os ruídos funcionam como interrogações da lógica do processo de coordenação na produção do cuidado. Cada trajetória-sentinela foi analisada a partir de um fluxograma, ou seja, de um diagrama-resumo que permitiu esquematizar os processos chaves que ocorreram, caracterizar o serviço estudado e identificar os ruídos gerados na assistência ${ }^{(3,9)}$. Para a construção dos fluxogramas analisadores foram utilizados os registros de prontuário (memória escrita), que documentam a passagem dos recém-natos pela unidade, e observação não participante das atividades da equipe de enfermagem, junto aos recém-nascidos participantes da pesquisa. A observação não participante teve como objetivo coletar dados para subsidiar a construção dos fluxogramas analisadores.

A segunda etapa envolveu a construção da rede de contratualidade da enfermagem com vistas à reflexão sobre seu papel e inserção, e as relações que estabelecem na UTIN. A rede de contratualidade expressa a complexa teia de (inter)relações entre atores sociais. A organização da rede é dada pela conexão entre os pontos (profissionais, serviços, setores, unidades de saúde) e seus padrões de interação; expressa, portanto, compromissos, expectativas, demandas, acordos, coalizões, solicitações, conflitos, tensões entre as partes. Compreender a dinâmica da conectividade significa compreender a rede.

A análise retrospectiva dos registros exigiu a ordenação cronológica dos fatos; identificação dos serviços envolvidos e das unidades assistenciais utilizadas; localização de resultados de exames, evoluções de enfermagem e pareceres médicos. Analisou-se o uso dos registros na tomada de decisões, em relação às condutas e definindo a lógica assistencial instaurada. A memória não-escrita de um caso foi resgatada através de uma entrevista semi-estruturada realizada com a mãe de um dos recém-natos estudados nesta pesquisa.

A rede de petição e compromissos foi construída através das discussões de grupos focais e marcada pelas contratualidades estabelecidas entre a equipe de enfermagem e os diversos atores sociais/serviços envolvidos na produção do cuidado ao recém-nato internado na UTIN. Participaram desta etapa da pesquisa 31 profissionais da equipe de enfermagem e um moderador (o próprio pesquisador) que pôde intervir para focalizar e aprofundar a discussão em torno do tema.

Os princípios éticos forma seguidos em todas as fases do estudo, em consonância com o que preconiza a Resolução 196/96. O estudo foi aprovado pelo Comitê de Ética em Pesquisa com Seres Humanos do Instituto Fernandes Figueiras sob o protocolo no 09/2008.

\section{RESULTADOS E DISCUSSÃO}

Foram acompanhados dois recém-nascidos, internados em uma UTIN, desde o período do nascimento até a alta hospitalar. O primeiro caso, denominado Caso 1, foi acompanhado durante os 64 dias de internação, período em que foram identificados 216 elementos ruidosos. O segundo caso, denominado Caso 2, foi seguido durante os 105 dias de internação do recém-nato; na descrição desta trajetória contou-se também com a entrevista do responsável pela criança. Durante o período de internação foram levantados 264 elementos ruidosos (Figura 1).
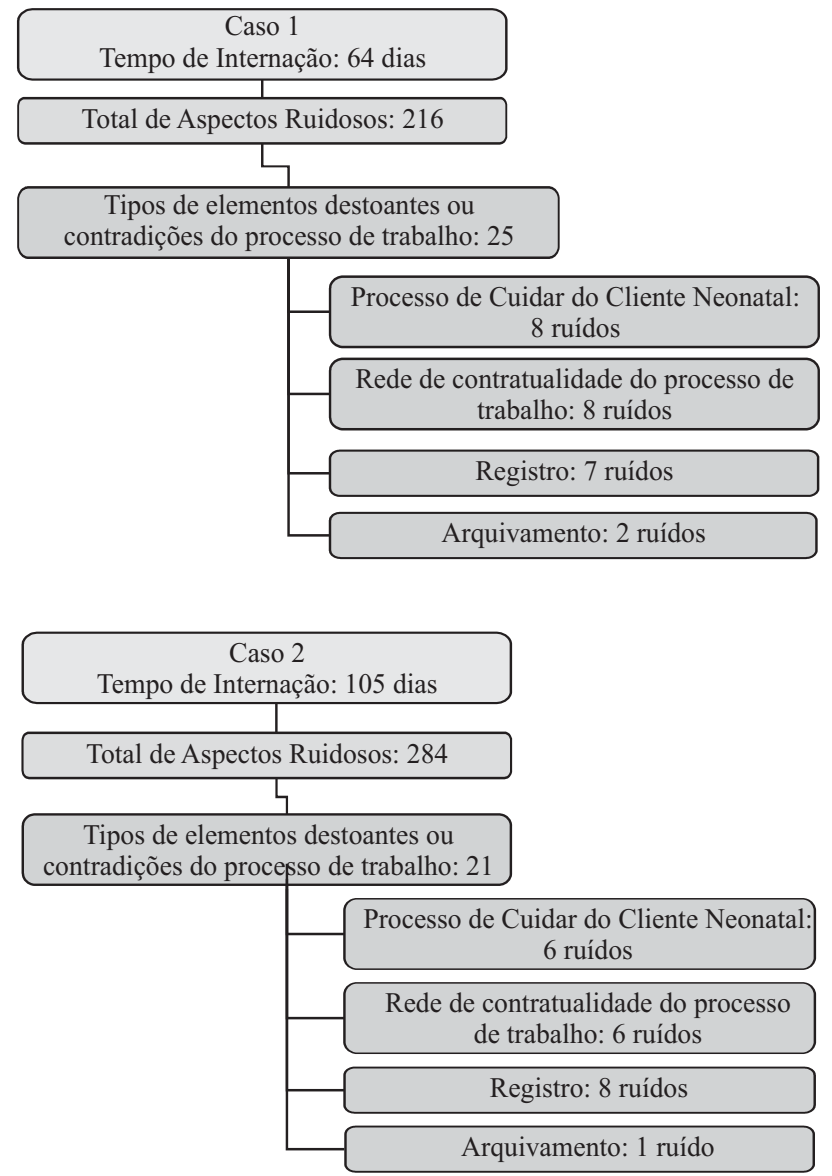

Figura 1 - Distribuição de ruídos entre as trajetórias sentinela, Unidade de Terapia Intensiva Neonatal da Maternidade estudada Rio de Janeiro - 2008

Da análise das trajetórias sentinelas, construídas a partir da leitura dos prontuários, foram apreendidos vários tipos de problemas, elementos destoantes no cotidiano assistencial que, de um modo geral, se repetiram ao longo do período de internação. Observou-se uma convergência entre os ruídos identificados através do estudo das trajetórias-sentinela (Figura 1) e da construção da rede de contratualidades. Foram identificados quatro grandes grupos de ruídos que envolveram: o cuidado ao recém-nato, a rede 
de contratualidade (relações) que se estabelece no desenvolvimento do cuidado, a qualidade dos registros e o arquivamento de documentos.

Os ruídos identificados foram classificados em interunidades e intraunidades ${ }^{(6)}$. Os ruídos interunidades se referem aos problemas que podem ser identificados quando algum serviço, produto e/ou insumo da unidade assistencial é solicitado ou consumido. Este tipo de ruído permite analisar as relações entre a unidade assistencial e seus fornecedores, ou ainda com outra unidade assistencial. Já os ruídos intraunidades são aqueles problemas que podem ser reconhecidos na própria equipe assistencial. Tais problemas interferem na qualidade da assistência prestada, uma vez que comprometem a integralidade da assistência. Os ruídos intraunidades também podem ser intra-equipe quando relacionados à falta de integração entre os profissionais que compõem o grupo de trabalho.

Destacaram-se como ruídos comuns entre os casos analisados (Figura 2):

1. Evolução médica e de enfermagem sem assinatura e/ou carimbo (Ruído Intra-equipe). Apesar de este ruído estar presente nos registros médicos, o problema se concentra principalmente entre os registros de enfermagem.

2. Registro da equipe de enfermagem (Ruído Intra-equipe). A equipe de enfermagem registra os cuidados realizados essencialmente no impresso destinado ao controle do balanço hídrico. Das anotações, somente as admissões e alguns procedimentos invasivos ou curativos são registradas pelos enfermeiros. O acompanhamento e a comparação do registro diário dos diversos profissionais responsáveis pela prestação de cuidados sugerem que algumas anotações deixaram de ser lidas/consideradas. A contradição entre avaliações e condutas descritas em alguns registros e a discussão no grupo focal concorrem para esta hipótese. Cabe destacar que se trata de uma observação pontual, restrita a alguns registros durante o período de seguimento dos casos.

3. Preenchimento incompleto das folhas de identificação do paciente (Ruído Intra-equipe). O não preenchimento ou o preenchimento incompleto dos cabeçalhos dos balanços hídricos, pela enfermagem, leva ao extravio, troca e a presença de balanços de homônimos (problemas de arquivamento).

4. Identificação de distermias sem a manutenção da temperatura na incubadora na zona termo-neutra, procedimento que favorece o controle da temperatura corporal do recém-nato (Ruído Intra-equipe).

5. Demora ou ausência de condutas em situações em que o recém-nato encontrava-se sem evacuar (Ruído Intraequipe).

6. Falta de articulação entre profissionais que assistem aos recém-natos (Ruído Intra-equipe). O fisioterapeuta, por exemplo, examina, evolui e indica condutas, mas não há indicativos no registro médico e da equipe de enfermagem que tais recomendações/condutas foram atendidas. O mesmo problema é identificado em relação ao Serviço de Fonoaudiologia.

7. Demora ou não atendimento do Serviço de Nutrição às solicitações (Ruído Interunidade - Unidade Assistencial/ Serviço de Nutrição). Tais ruídos foram identificados naquelas situações em que o Serviço de Nutrição, apesar de ser previamente contatado pela equipe de enfermagem, não enviou a dieta adequadamente. $O$ fato compromete o ganho de peso do recém-nato internado na UTIN.

8. Demora na realização de exames (Ruído Interunidade).

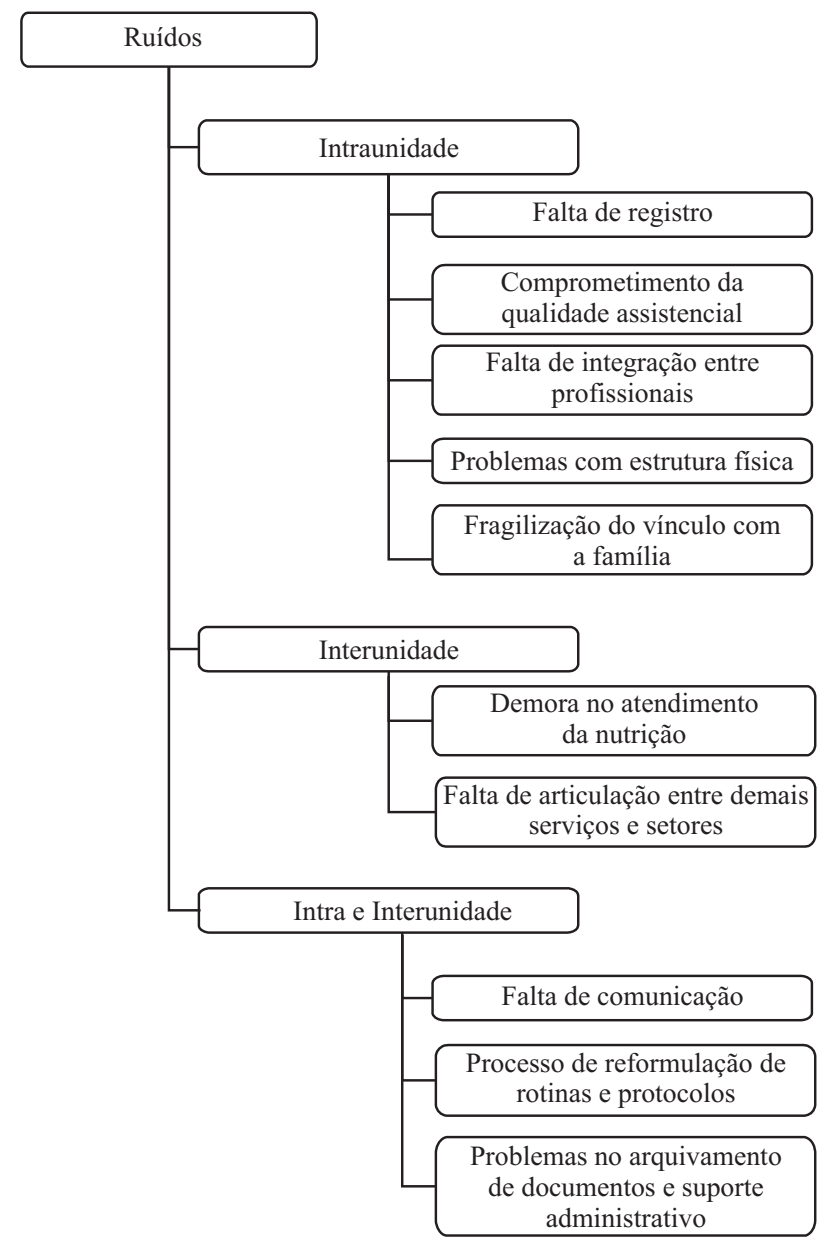

Figura 2 - Distribuição dos ruídos identificados a partir das trajetórias-sentinela e dos grupos focais, Unidade de Terapia Intensiva Neonatal da Maternidade estudada - Rio de Janeiro - 2008

O estabelecimento de vínculo entre o responsável pelo recém-nato e a equipe está entre os ruídos específicos que chama mais atenção (ruído intraunidade). Na entrevista a mãe de um dos recém-natos acompanhados relata que se sentia excluída pela equipe médica quanto à participação na assistência prestada ao seu bebê. Isso ocorria especialmente durante a visita diária (round). A mãe diz se sentia invisível neste momento, pois ficavam de costas falando 
do meu filho. Em uma dessas visitas a mãe relata que interveio e questionou a postura dos médicos. Relata que os médicos por sua vez nada declararam, mas não ficaram mais de costas; no entanto continuava me sentindo excluída deste processo.

A realização do grupo focal permitiu o levantamento e discussão dos ruídos que interferem na assistência prestada e na construção da rede de contratualidade, favorecendo o debate do grupo a cerca do papel e inserção da equipe de enfermagem, das relações e da gestão do cuidado. $\mathrm{Na}$ construção desta rede emergiram pontos em comum com os fluxogramas das trajetórias sentinelas e foram identificadas tensões/conflitos no dia-a-dia dos profissionais.

Dos ruídos identificados através do grupo focal, alguns vêm iluminar a análise sobre o processo de trabalho: falta de comunicação entre as equipes multidisciplinares, principalmente entre a equipe de enfermagem, médicos, fisioterapeutas, fonoaudiólogos, Serviço de Nutrição e Farmácia; falta de articulação interunidades; comprometimento da qualidade assistencial; necessidade de (re)formulação de rotinas e protocolos; problemas relacionados a registro e arquivamento de documentos; fragilização do vínculo com a família.

O processo assistencial é centrado na figura do médico e há uma participação restrita dos demais profissionais da equipe no projeto terapêutico, definido por uma categoria profissional específica. Verifica-se um compartilhamento interdisciplinar restrito e falta de integração e articulação entre profissionais. Isolado de um trabalho mais coletivo e interdisciplinar no interior da equipe, este desenho assistencial gera uma dimensão cuidadora pouco comprometida com seu sujeito/objeto de trabalho - o cliente. Os elementos essenciais para qualificar os atos de saúde - vínculos, responsabilização e acolhimento - são muito pouco trabalhados na relação entre os profissionais de saúde e o cliente.

A comunicação é insuficiente e realizada predominantemente através do prontuário. Problemas com os registros em prontuário foram evidenciados nos fluxogramas e no relato dos grupos focais. Os registros são lidos parcialmente, o que interfere sobremaneira no processo assistencial. A equipe de enfermagem não demonstra utilizar bem os espaços de negociação.

Apesar da complexidade desta unidade especializada, há problemas na sistematização do cuidado de enfermagem e na evolução diária da enfermeira nos prontuários. Sobre o trabalho da enfermeira, na discussão dos grupos focais, os participantes justificam como falta de tempo destes ruídos. Afirmam que atividades do cotidiano (comandar a equipe de enfermagem, prever, prover, organizar e controlar os insumos necessários), limitariam suas ações e participação no projeto terapêutico. Apesar de a enfermeira compartilhar de uma rede de contratos e significações ${ }^{(10)}$, permanecem expostos problemas inerentes ao saber-fazer da enfermagem e aos mecanismos de reação ao que está, aparentemente, estabelecido.
Destaca-se a fragilidade de algumas das redes de contratualidade estabelecidas no interior da unidade estudada, impedindo que sejam consolidados os acordos necessários às relações entre setores ou serviços e produzindo distorções no processo assistencial. A fragilidade na contratualização tem sido uma característica comum a várias instituições, verificada em outros estudos ${ }^{(3-4,11-12)}$. O sobre-esforço resultante destas distorções muitas vezes gera o retrabalho, já que boa parte do tempo dos profissionais é consumindo na cobrança dos serviços solicitados e na superação de dificuldades encontradas no dia-a-dia da prestação do cuidado. De um modo geral tais redes são construídas de forma espontânea, extremamente dependente das relações interpessoais existentes e de mecanismos informais de comunicação.

O processo de trabalho é fragmentado e os projetos não são, a princípio, explicitados e nem negociados adequadamente, de forma a garantir a integralidade da assistência. Tal contexto mostra a necessidade de mobilização de estratégias que fortaleçam o trabalho em equipe, com o foco na integralidade do cuidado e satisfação das necessidades do cliente. Tal fragmentação, fruto de um processo de trabalho cada vez mais especializado e com grande incorporação tecnológica, significa em parte restrição e diferenciação da assistência, redução na produção assistencial, custos mais elevados, discriminação e seleção da clientela ${ }^{(3,6)}$.

Identifica-se uma estrutura administrativa burocrática e um cenário formado por diversos grupamentos de indivíduos (equipe multiprofissional), que disputam seus projetos. Neste cenário reside a predominância do poder e da autonomia médica.

A falta de integração e articulação entre os profissionais representa um elemento gerador de tensões, expresso através de uma série de dificuldades na realização do trabalho em equipe. Os espaços de compartilhamento (reuniões clínicas, round diário) não se efetivam com tal. Nesse trabalho coletivo, a autonomia dos profissionais nãomédicos (nutricionistas, fisioterapeutas, enfermeiros) é relativa. Condutas traçadas por estes profissionais eventualmente não são seguidas e seus registros desvalorizados, ainda que sinalizem algum tipo de anormalidade não identificada pelo corpo médico. Os profissionais não-médicos estão presentes, mas não integram ou consubstanciam o processo de gestão do cuidado.

A análise dos fluxogramas e das discussões nos grupos focais revela que as recomendações do Serviço de Fisioterapia e de Fonoaudiologia não são acolhidas/seguidas pela equipe médica e de enfermagem; segundo um dos participantes do grupo focal é como se a visita deles não existisse. Como conseqüência há tensões no processo gerencial do cuidado. No processo assistencial, o corpo médico coordena, mas não está presente integralmente; a enfermagem está presente, mas não é reconhecida como gestora ou não se reconhece como tal. Nessa estrutura os demais profissionais são solicitados de acordo com a necessidade do cliente, mas não estão inseridos no projeto assistencial e na efetivação do cuidado. 
A assistência está centrada na figura do médico que tem plena autonomia nas decisões e controla o processo de trabalho na UTIN como um todo. A organização tecnológica e do trabalho favorecem ações fragmentadas, impedindo que o trabalho em equipe se efetive como tal através do planejamento coletivo da assistência e discussão multidisciplinar. Na UTIN, o trabalho médico tem como elemento central o processo de diagnóstico e a terapêutica. Uma vez que o compartilhamento interdisciplinar é restrito, dificuldades inerentes ao cotidiano assistencial geram contradições e ações pouco articuladas onde cabe a uma categoria profissional específica a palavra final(13-14).

Verificou-se no estudo tempos de internação muito elevados (acima de 60 dias). Comumente utilizado como um indicador de eficiência hospitalar e medida substitutiva de custos, o tempo de permanência tem sido visto como um indicador relacionado à qualidade do cuidado prestado(15), embora a natureza desta relação ainda permaneça obscura. A avaliação do tempo médio de permanência deve levar em conta as características dos pacientes e do hospital, tipo de remuneração da internação e disponibilidade de tratamentos alternativos, como o tratamento domiciliar, por exemplo. Tempos de permanência significativamente mais longos do que o esperado podem ser vistos como indicativo de ineficiência administrativa ou baixa qualidade do cuidado prestado, visto que esta permanência pode ser necessária em decorrência de complicações resultantes do cuidado deficiente. Ruídos inter e intra-unidades contribuem indiretamente para o aumento do tempo de internação.

A análise do processo de trabalho revelou obstáculos que interferem na qualidade do cuidado prestado, gerando sombras e contradições nem sempre identificadas pelos gestores e pelos profissionais de enfermagem imersos em seu cotidiano assistencial. A análise do processo de trabalho pode possibilitar a abertura da caixa preta que envolve os processos de produção do cuidado, permitindo o desvelamento da dinâmica dos modelos tecnológicos da saúde. Para tanto é necessário ultrapassar barreiras, especialmente aquelas referentes ao campo das relações. A equipe de enfermagem da UTIN revelou grande disposição em explicitar essas tensões e rever contratualidades.

\section{REFERÊNCIAS}

1. Krauss-Silva L. Avaliação tecnológica em saúde: questões metodológicas e operacionais. Cad Saúde Pública. 2004;20 Supl 2:S199-207.

2. Bosi MLM, Uchimura K. Avaliação da qualidade ou avaliação qualitativa do cuidado em saúde? Rev Saúde Pública. 2007;41(1):150-3.

3. Merhy EE, Onocko R, organizadores. Agir em saúde: um desafio para o público. São Paulo: Hucitec; 2007.
Enfrentar os nós críticos que afetam o processo de trabaIho requer valorizar recursos de diversas ordens e exige a participação de todos os profissionais que se responsabilizam pelo acompanhamento do cliente internado. Neste sentido, garantir a integralidade da assistência pode envolver novas configurações das redes estabelecidas e (re)construção das relações no interior das equipes. Há que se resgatar/formar vínculos entre as equipes de trabalho e interagir saberes e fazeres.

Acredita-se que o estímulo ao trabalho cooperativo e às ações e movimentos instituintes crie um ambiente favorável à construção do novo, com base em um projeto assistencial construído coletivamente voltado para a resolução das necessidades do cliente ${ }^{(16)}$.

\section{CONCLUSÃO}

Ao reconhecer os trabalhadores de saúde como sujeitos do processo e com o poder de intervenção e implementação de projetos, esse estudo viabilizou a identificação dos problemas que interferem na UTIN e que, conseqüentemente, afetam a qualidade da assistência prestada. A efetividade do cuidado passa pelo necessário desafio de equilibrar a tensão existente entre o conceito amplo e multidimensional de valorização da vida, de aperfeiçoamento técnico-científico dos profissionais de saúde, de desenvolvimento tecnológico e do uso racional de recursos existentes na instituição.

A trajetória-sentinela, dispositivo analisador da qualidade de assistência, permitiu avaliar conjuntamente todo processo assistencial e resultados, sinalizando ruídos ao longo do acompanhamento dos casos, momentos e formas de operacionalização do trabalho vivo, da produção do cuidado - objeto dessa pesquisa.

Para que a análise e discussão das tensões reveladas no fluxograma sejam proveitosas é necessário ultrapassar barreiras relacionadas à comunicação. A quebra do silêncio do cotidiano desvela a presença de processos instituintes que não haviam sido pensados pelo modelo de organização e gestão do equipamento institucional, bem como abre possibilidades de interrogações sobre a maneira como se opera o processo de trabalho e o sentido de suas ações naquele equipamento.

4. Franco TB, Merhy EE. O uso das ferramentas analisadoras para apoio ao planejamento dos serviços de saúde In: Merhy EE, Magalhães Junior HM, Rimoli J, Franco TB. O trabalho em saúde: olhando e experienciando o SUS no cotidiano. São Paulo: Hucitec; 2004. p.135-60.

5. Morin E. Sociologia: do microssocial ao macroplanetário: revisada e aumentada pelo autor. Lisboa: Publicações Europa-América; 1998. 
6. Jorge AO. A gestão hospitalar sob a perspectiva da micropolítica do trabalho vivo [tese doutorado]. São Paulo: Faculdade de Ciências Médicas, Universidade Estadual de Campinas; 2002.

7. Kamada I, Rocha SMM. As expectativas de pais e profissionais de enfermagem em relação ao trabalho da enfermeira em UTIN. Rev Esc Enferm USP. 2006;40(3):404-11.

8. Minayo MCS. O desafio do conhecimento: pesquisa qualitativa em saúde. 7ạ ed. São Paulo: Hucitec; 2000.

9. Barboza TAV, Fracolli LA. A utilização do "fluxograma analisador" para a organização da assistência à saúde no Programa Saúde da Família. Cad Saúde Pública. 2005;21(4):1036-44.

10. Tonini T. Enfermeira instituída/instituinte: a subjetividade das estratégias de cuidar [tese doutorado]. Rio de Janeiro: Instituto de Medicina Social, Universidade Estadual do Rio de Janeiro; 2006.

11. Marques GQ, Lima MAD. Organização tecnológica do trabaIho em um pronto atendimento e a autonomia do trabalhador de enfermagem. Rev Esc Enferm USP. 2008;42(1):41-7.
12. Ribeiro EMPD, Blank VLG. A teorização sobre processo de trabalho em saúde como instrumental para análise do trabalho no Programa Saúde da Família. Cad Saúde Pública. 2004; 20(2):438-46.

13. Gaiva, MAM, Scochi, CGS. Processo de trabalho em saúde e enfermagem em UTIN. Rev Lat Am Enferm. 2004;12(3):469-76.

14. Duarte ED. O cuidado ao RN na perspectiva da integralidade: saberes e práticas no cotidiano de uma UTIN [tese doutorado]. Belo Horizonte: Universidade Federal de Minas Gerais; 2007.

15. Noronha MF, Portela MC, Lebrão ML. Potenciais usos dos APDRG para discriminar o perfil da assistência de unidades hospitalares. Cad Saúde Pública. 2004;20 Supl. 2: S242-55.

16. Franco TB. Fluxograma descritor e projetos terapêuticos para análise de serviços de saúde, em apoio ao planejamento os casos de Luz (MG) In: Merhy EE, Magalhães Junior HM, Rimoli J, Franco TB. O trabalho em saúde: olhando e experienciando o SUS no cotidiano. São Paulo: Hucitec; 2004. p.160-98.
Correspondência: Enirtes Caetano Prates Melo

Rua Dr. Xavier Sigaud, 290 - $3^{\circ}$ andar - sala 308 - Praia Vermelha CEP 22290-180 - Rio de Janeiro, RJ, Brasil 